\title{
Review
}

\section{Oral Health for Infants: What Pediatricians and Pediatric NeoNatal Nursing Staff Need to Know}

\author{
John E. Nathan, DDS, MDS* \\ Department of Pediatric Dentistry, University of Alabama, Birmingham and Case Western Reserve University, Cleveland, OH, USA; Department of Otolaryngology and Dentistry, \\ Northwestern University, Feinberg School of Medicine, Chicago, USA
}

"Corresponding author

John E. Nathan, DDS, MDS

Adjunct Professor, Department of Pediatric Dentistry, University of Alabama, Birmingham and Case Western Reserve University, Cleveland, OH, USA;

Clinical Associate Professor, Department of Otolaryngology and Dentistry, Northwestern University, Feinberg School of Medicine, Chicago, USA

E-mail: jnathandds@gmail.com

\section{Article information}

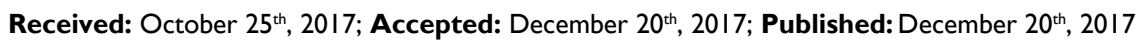

\section{Cite this article}

Nathan JE. Oral health for infants: What pediatricians and pediatric neonatal nursing staff need to know. Pediatr Neonatal Nurs Open J. 20I7; 5(I): II-18. doi: 10.17I40/PNNOJ-5-128

\section{| ABSTRACT |}

This manuscript focuses on relevant and fundamental aspects of oral healthcare of the newborn and infant for the pediatrician and nursing personnel. In almost all cases, these medical providers have first contact with babies and infants during the first year of life and rarely does medical curricula include time or opportunity to provide exposure to fundamentals of oral health care. As such it becomes important to provide pediatricians and neonatal nursing personnel with what will enable optimal oral health and early recognition of oro-facial normalities $v s$. abnormalities, relevant risk assessment and management of dental caries, enamel and tooth malformations, and common oral pathology. Discussion is primarily directed at issues that affect the newborn and early toddler until which time that a relationship is established and a dental home with a pediatric dental specialist is identified.

\section{Keywords}

Pediatrician; Healthcare; Nursing.

\section{Abbreviations}

AAP: American Academy of Pediatrics; AAPD: American Academy of Pediatric Dentistry; ECC: Early Childhood Caries; ANUG: Acute Necrotizing Ulcerative Gingivitis.

\section{INTRODUCTION}

W hat might surprise most healthcare providers is the fact that the most frequently encountered chronic disease of children is dental caries. It wasn't long ago that the recommendations of the American Academy of Pediatrics (AAP) for much of their long history recommended that the child first seek dental evaluation by three years of age. ${ }^{1}$ This reflected the general belief that child behavior under three years of age rendered limited usefulness to early examinations and secondly that there were inadequate numbers of pediatric dental specialists available to see children earlier. In contrast to views of pediatric dentists, those responsible for treating extensive early childhood decay it became clear that deferral to age three for first seeing a dentist was clearly not in the best interest of children and parents. Since the formulation of the specialty of Pedodontics (today referred to as Pediatric Dentistry) recognition of the high demand for invasive repair of multiple and extensive decay among children from age 1 to three, the American Academy of Pediatric Dentistry (AAPD) has long advocated institution of earlier preventive measures than three years.

Enhanced bipartisan interaction of the AAPD with its medical counterpart, the AAP, over the course of the past decade has modified its periodicity schedule to suggest seeking a dental home for children before the child's first birthday. ${ }^{2}$ It remains a fact today that there are approximately 10 pediatricians for every pediatric dentist nationally and access to care for pediatric dental expertise is not universal, let alone uniformly sought before 12 months of age. While greater numbers of general dentists exist to see infants, few are trained or comfortable with examination, diagnoses, or treatment of newborns. Responsibility for diagnostic or interventional care falls largely on healthcare providers who have 
earliest exposure during the first year of life, i.e., pediatricians, family practice physicians, and neonatal nursing personnel. As such, early inspection, even if conducted optimally, need include caries risk assessment, assessment of genetic and acquired anomalies, appropriate preventive measures and fluoride supplementation. Responsibility for education and instruction of new parents to available hygiene procedures and their frequency falls largely on nursing personnel.

While diagnosis of early caries, oro-facial abnormalities, or variations of normality is not expected from medical personnel, they remain in an optimal position to determine when referral and intervention are warranted. The global objective of this paper is to provide basic insight and exposure to key elements of diagnosis and interception of dental disease and abnormality for the pediatrician and nursing personnel during the natal and neonatal period.

Issues are discussed which represent common findings amongst newborns and early infancy. They include teeth found to erupt prematurely (neonatal or natal teeth), supernumerary teeth, soft and hard tissue anomalies including Riga Fede's Disease, gingivitis and soft tissue pathology, ankyloglossia, enlarged frenulum, abnormalities in tooth emergence and eruption, Bohn's nodules (Epstein's Pearls), eruption hematomas and cysts, defects in enamel and dentin formation, premature exfoliation of primary teeth, oro-facial traumatic injury, bruxism, and early management of dental habits and resultant deformation of alveolar structures, and classification of malocclusions in the early primary dentition. The scope of this paper becomes limited and not all commonly occurring and variable normality and abnormalities can be included.

The impact of early feeding habits inclusive of breast feeding, nursing bottle habits, diet and parental oral hygiene will receive significant attention. Early childhood caries (ECC) despite a complete awareness of its course, etiology, and available prevention strategies is amongst the most common of causes of need for extensive dental restorative and surgical care in infancy often necessitating general anesthetic. This dental topic receives the most attention during infancy, but its overwhelmingly high incidence encountered across all socioeconomic strata remains puzzling other than to conclude that preventive strategies are clearly not reaching these young parents and families. Lastly, perhaps as confusing as that is, there is probably greater misinformation and miscon- ception concerning the beneficial effects and appropriateness of fluoridation. Effort will be directed to clarify misconceptions and indications for the proper use of fluoride in both water supplies and daily supplementation.

\section{NATAL AND NEONATALTEETH}

While eruption patterns for most infants suggest the first tooth emerges at approximately 4-6 months of age, a very small percentage of infants have manifested the presence of what appears to be an early emerging primary lower central incisor. Differential diagnosis includes: 1) prematurely erupting actual central incisor, or 2) Emergence of an "extra" tooth. Sometimes the tooth can be covered partially or completely with mucosal tissue, and gives the appearance of being highly mobile, through or under gingival tissue.

Clinical concerns exist when this tooth manifests significant mobility to the extent that exfoliation, should it occur, poses a problem for potential aspiration and airway obstruction. Whether an extra tooth or the actual early erupted primary central incisor, these emerge with varying degrees of mobility due to immature or no root formation. A dental intra-oral X-ray should be taken to determine if potential exists to maintain the tooth as root formation occurs. If an extra tooth, extraction is recommended; if an actual incisor, airway concerns override retention concerns (Figures 1 and 2).

\section{RIGA FEDE'S DISEASE}

In rare instances early eruption of a lower incisor has potential to create a benign, ulcerative granulomatous lesion on the ventral surface of the tongue. The superior edge of the tooth may show developmental ridges called mamelons that may be exaggerated and coarse to irritate the underneath side of the tongue. This is not truly a "disease," but can be irritating for these infants. If erosion in the ulcerative area is severe, the incisal edge of the tooth may be smoothed; the problem usually becomes self-limiting (Figure 3).

\section{EARLY GINGIVITIS (Mild, Moderate, and Severe)}

Infants, small children, adolescents and adults are susceptible to the presence and retention of dental plaque if permitted to remain around the necks of erupted teeth. Brushing of all tooth surfaces
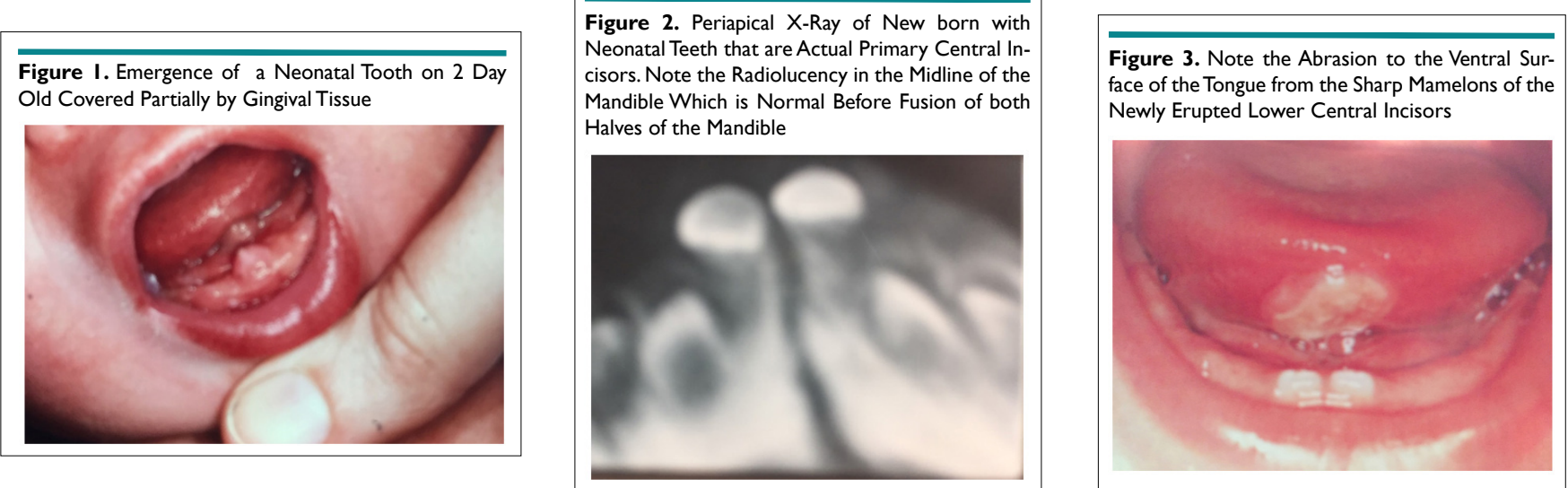
against the upper incisors, these sounds are difficult to make. That said, judgment is needed to differentiate those who need surgical resection vs those who simply overcome the anatomical limitation. In any event, surgical intervention can readily be accomplished when indicated by a pediatric dentist, oral and maxillofacial surgeon, or ear-nose-throat (ENT) surgeon using a surgical scissors, soft tissue laser, or scalpel. Frequently surgical closure is needed with reabsorbable suture placement. Often use of soft tissue lasers can serve to minimize or eliminate this need (Figure 7).

A similar but unrelated anatomical variation can be observed in the anterior maxillary vestibule of some infants where a tissue redundancy can occur between the upper lip and the upper deciduous incisors extending to the anterior dental papilla as seen below. This is generally of no consequence and only becomes of interest when the tissue interferes with alignment of the permanent central incisors at 10-11 years of age. Orthodontic treatment involving closure of the diastema precedes surgical excision of the frenulum for best results. It is not an uncommon occurrence that infants and toddlers fall in these early years and potential exists to experience a traumatic tear of this attachment. Considerable bleeding results and is of great parental concern, but easily managed with the problem essentially eliminated (Figure 8 and 9).

\section{TOOTH ERUPTION VARIATIONS AND ABNORMALITIES |}

Bohn's Nodules (aka Epstein's Pearls) are connective tissue bulges usually composed of keratin which are generally covered by oral mucosa in anterior regions where maxillary or mandibular anterior deciduous incisors will eventually emerge. These may be frequently seen in newborns and are often mistaken for erupting tooth buds. They resolve spontaneously and require no intervention (Figures 10, 11 and 12).

\section{ERUPTION HEMATOMAS}

These are characterized by bluish-discolorations and sometimes swellings in anterior or posterior alveolar ridge when and where primary incisors or molars are expected to emerge. They generally have appearance of subgingival bleeding around a developing and emerging tooth that appears to be having difficulty erupting. Invariably, these resolve spontaneously and require no intervention (Figure 13). minimizes the ability to enunciate "th," "ph," and "l," sounds. Because of an inability to extend and push the tip of the tongue up
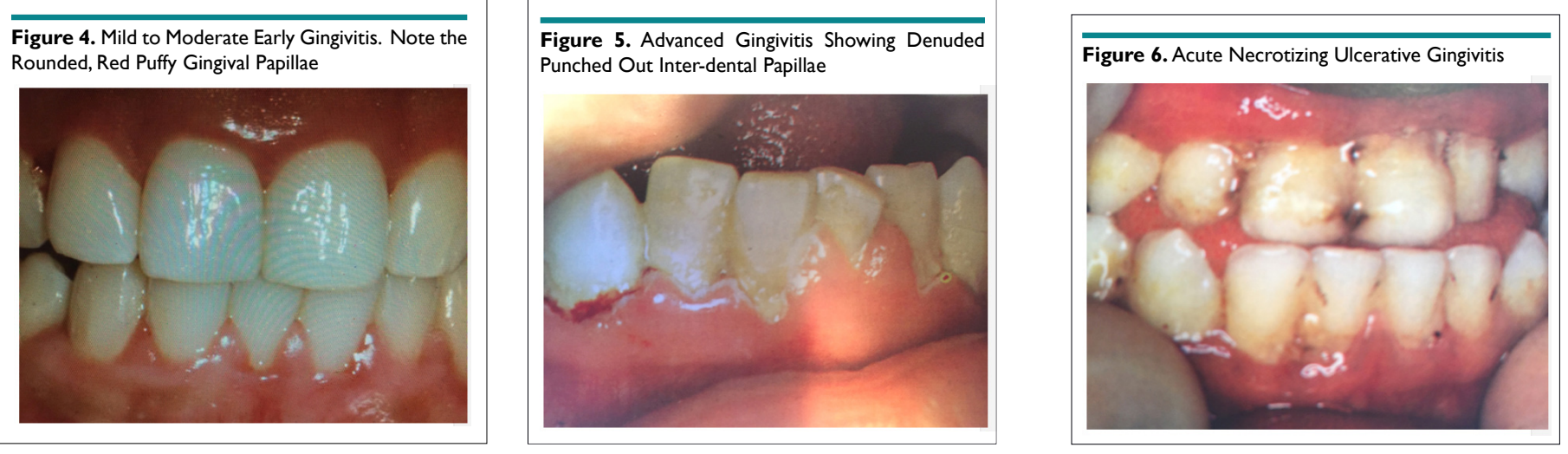


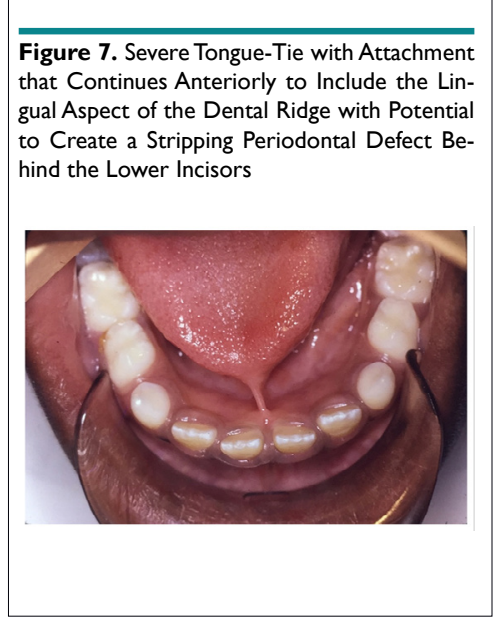

Figure 10. Epstein's Pearl on New Born. Also Seen in the Midline of the Palate at the Junction of the Hard and Soft Palate
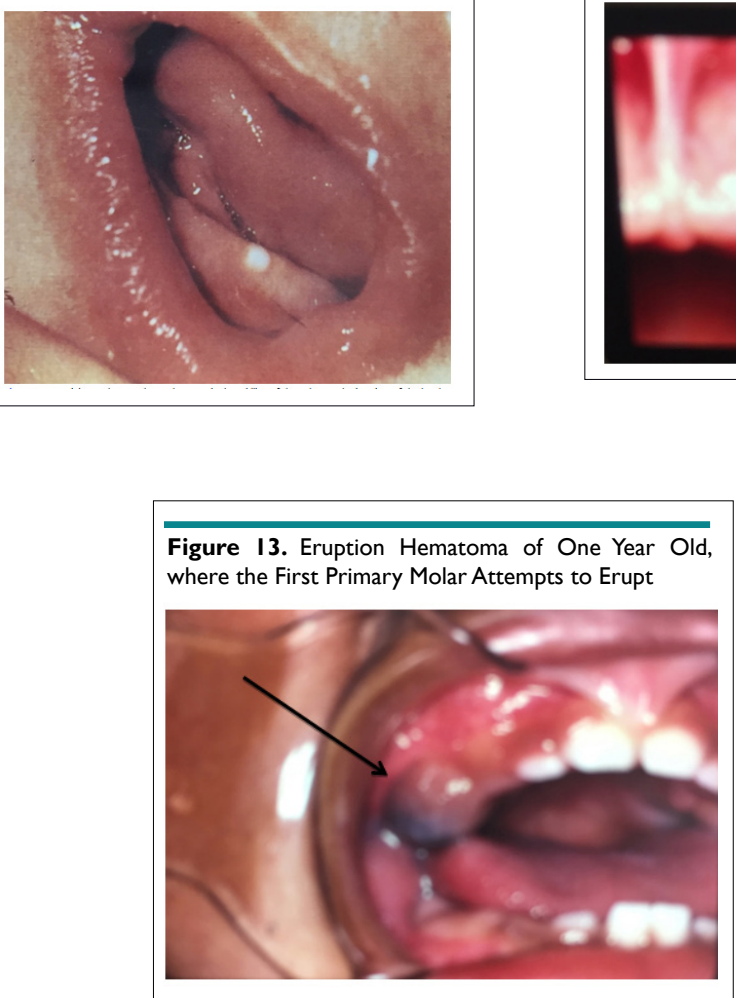

\section{ERUPTION CYSTS}

Eruption cysts are associated with permanent teeth which can offer the same presentation as eruption hematomas; difference is these are frequently encapsulated connective tissue and may require surgical excision and exposure of the permanent tooth. Not usually an issue for children under age 6 years (Figure 14).
Figure 8. Nine Months Old Infant with Clinically Insignificant Enlarged Frenulum. Unlike the Tongue-Tie, Surgical Management of this Frenulum is Unnecessary Until Age II

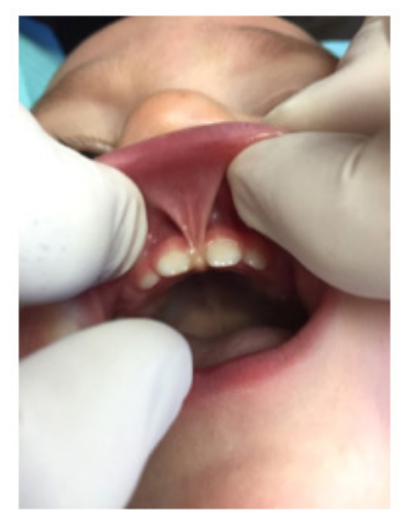

Figure I I. Bohn's Nodule, Keratin Cyst on 2 Months Old on the Alveolar Ridge in Regions where Teeth will Later Emerge

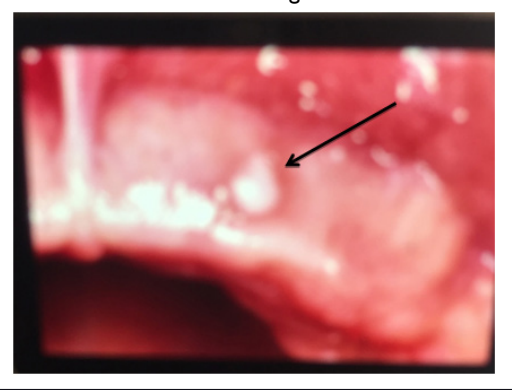

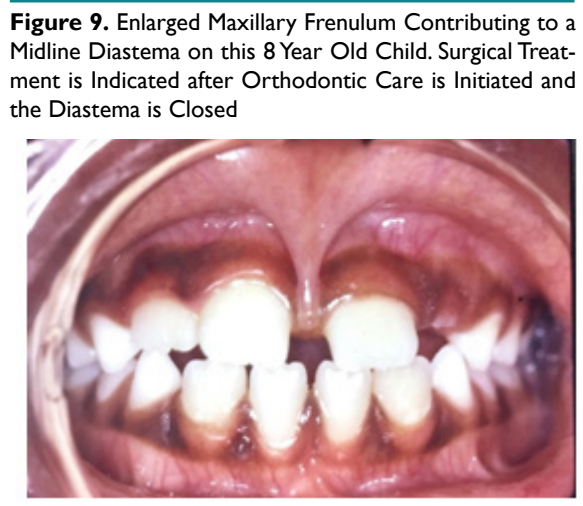
Midline Disstema on this 8 Year Old Child Surgical Treat-

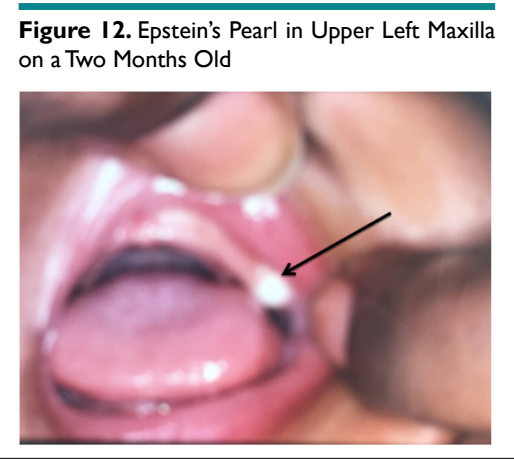

Figure 14. Eruption Cysts Covering the Emerging Permanent Lateral Incisors Often Requiring Surgical Excision

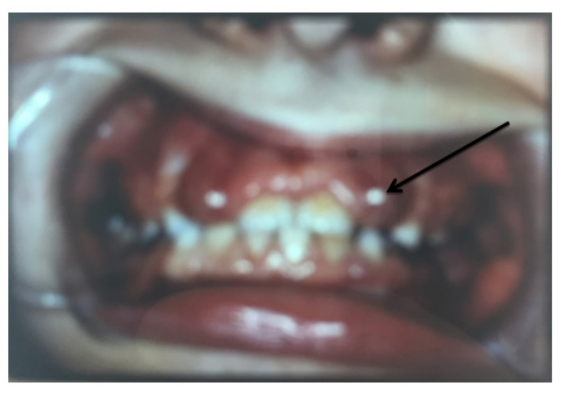

\section{EARLY PREVENTION - WHEN AND HOW TO BRUSH INFANT'S TEETH}

In the presence of twice daily tooth brushing, early dental decay (caries) is unlikely. Tooth brushing by parents or nursing care personnel is unnecessary till the first tooth begins to emerge. 
While some believe it advantageous to brush the tongue to clear and remove debris from its deep crevices, when teeth are present or not, there is no evidence to suggest this is productive. Dental caries can occur on any one surface of a tooth, or multiple teeth distributed where plaque is not removed by adequate parental brushing.

Any method that removes plaque (bacteria) accumulation, residual milk or formula, or food from the surfaces of the teeth is the goal of brushing. Recommended during the first year is the use of a soft-bristled toothbrush moistened with water. Toothpaste, with or without fluoride is not essential, despite manufacturers' claims of value. Mechanical toothbrushes where bristles rotate or vibrate in some manner are useful.

Position of the patient during brushing: Any position that permits visibility of the area to be brushed is appropriate. Supine position on the changing table contributes to parental access and visibility. Knee-to-knee positioning of parents, with the infant's head in the lap of the parent performing the brushing is generally ideal; in this position, the parent assisting can gently hold the infant's hands and facing the infant can distract or entertain the infant while the other parent accomplishes the task of brushing. Using a speck of toothpaste without fluoride can be used and this amount should be sufficiently small that the toothpaste neither obstructs the parents' visibility nor causes gagging of the infant. Given the small amount of toothpaste used, the infant can then safely swallow the saliva and toothpaste. It is noteworthy that while some 65 or so toothpastes are on the market; there is no hard evidence that any one is better than another; they contain the same components and with the exception of taste and texture, they are essentially identical to one another. Some contain baking soda and hydrogen peroxide which are generally intended for older children/adults. If there is a critical timing involved, it is generally considered after first and last feedings of the day. Additional brushing before bedtime is optimal, but not easily accomplished if the infant finds brushing annoying if not upsetting. While some infants readily permit this type of oral stimulation, there are others who resist every effort. Placing the infant to bed directly after feeding be it breast feeding or bottle feeding with a decay-promoting substance, (milk, formula, or sugar solution) or leaving the bottle with the infant to initiate sleep is to be discouraged and avoided whenever possible. When used as a pacifier or method to induce sleep, exposure to cariogenic fluids at this juncture is prone to early development of potentially extensive dental decay. At night, even babies do not salivate much; leaving sugar in contact with tooth enamel in an otherwise dry mouth likely places the child at risk to ECC (Figure 15, 16 and 17).

\section{EARLY CHILDHOOD CARIES (AKA ECC, NURSING MOUTH SYNDROME, BABY BOTTLE DECAY) |}

ECC is an entirely preventable phenomenon. It occurs on both breast fed and bottle fed infants. Those placed to bed with a bottle, or have access to on demand breast feeding without regular brushing, are the most susceptible. Regrettably, thousands of infants experience this annually in the U.S.; many require surgical center or hospital admission for general anesthetic to repair and /or remove multiple and extensively decayed teeth. Distinguishing features of this entity are rapid enamel and tooth destruction limited to upper (maxillary) teeth, most frequently the upper four incisors and first primary molars. Lower teeth are usually spared because the breast or bottle nipple rests on top of the tongue and the tongue essentially protects the lower teeth. Decay of lower anterior deciduous teeth is in general quite rare; when seen, it is generally the result of a complete absence of tooth brushing. That said, there are infants who despite breast feeding or bottle feeding habits or an absence of parental brushing, escape all forms of early decay. We refer to these infants as "LUCKY." There fortunately are those in the population that for reasons which remain unclear, are immune to dental decay.

For infants identified as "UNLUCKY" and situations where parents experience colicky infants and those who find breaking the breast or bottle feeding habit challenging, frustrating, or impossible, it is recommended that infants be weaned off the bottle, with gradual dilution of the bottle contents to eventually contain only water. For example, the first three days, dilute the milk or formula to three quarters milk or formula, the next three days half water/ half formula, three more days, three quarters water, and so on till the bottle contains only water. Easier said than done for breast fed infants! Preparation for some hard nights ahead is
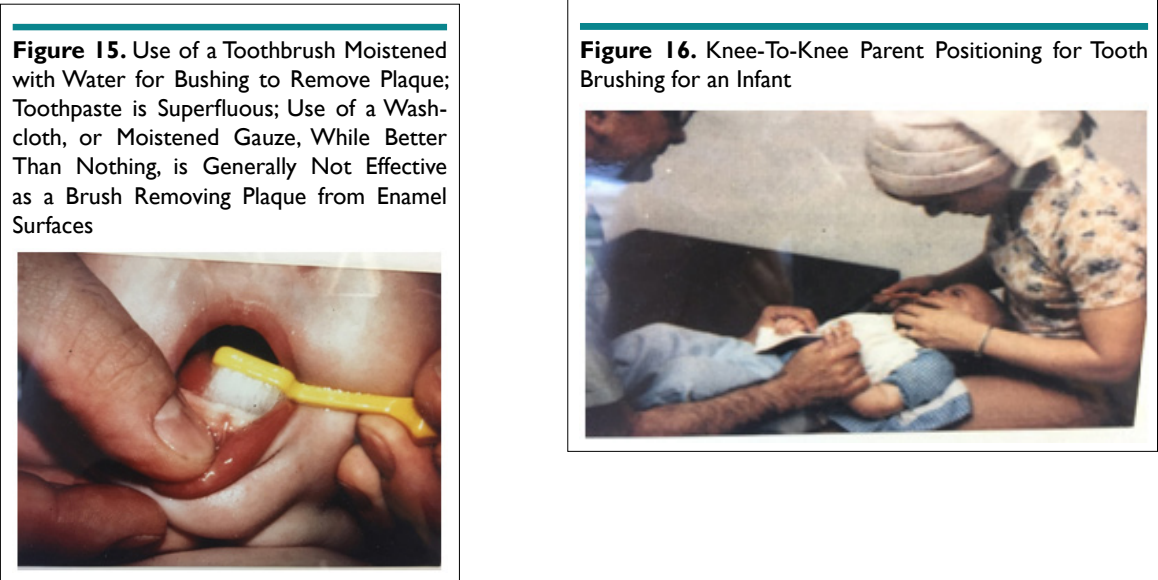

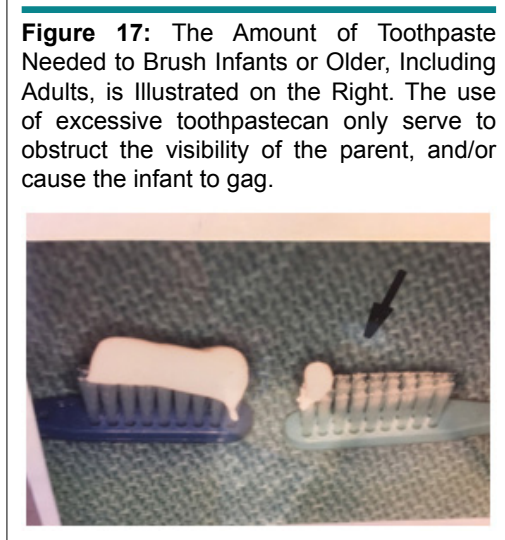


probable....

Figures 18, 19 and 20 are clinical photos of variations of ECC and commonly occurring decay for the purpose of early diagnosis and recognition.

\section{Early, Moderate, and Advanced Dental Decay in Young Children}

The anatomical depth of grooves in teeth other than front incisors can serve to trap bacteria (plaque) and initiate dental decay, despite good brushing habits. Examples of early, moderate, and advanced decay involving posterior teeth are shown in the below clinical photographs (Figures 21-28).

\section{FLUORIDE AND INDICATIONS FOR SUPPLEMENTATION -}

The sole intent of fluoride is to reduce the incidence of decay. While the mechanism by which this is accomplished has been extensively studied, it remains somewhat unclear how fluoride, absorbed in to the surface of tooth enamel renders the enamel less susceptible to acid given off by oral bacteria. Nevertheless, considerable research dating back to the 1940's found that the addition of fluoride to communal water supplies alone resulted in significant reductions in caries incidence. Optimal water fluoridation has been found to have 1 part per million (ppm). ${ }^{3}$ Infants do not need fluoride supplementation until 6 months of age. For those whose diets are limited to breast feeding or infant formulas using either non-diluted formula or diluted formula with non-fluoridated water, supplementation is warranted after 6 months of age. Increas- ing dosage needs depend on caries risk and age guidelines readily available through the AAP and AAPD. ${ }^{4}$

If a little fluoride is good, too much fluoride is unhealthy to the extent that excessive fluoride can cause permanent discoloration of permanent teeth which begin calcification at birth. These discolorations are termed "Fluorosis" and are observed when water supplies contain from 2-4 times recommended levels of fluoride. While the appearance of critics of fluoride continue to appear periodically, there is no viable evidence that fluoride when used properly, contributes to or causes cancer, liver disease, bone, muscular or other diseases and is without merit.

There is no scientific evidence that fluoride given prenatally is of benefit (Table 1).

\section{DEVELOPMENTAL ANOMALIES IN ENAMEL AND DENTIN FORMATION}

\section{Amelogenesis Imperfecta}

Hereditary defect in which enamel formation is adversely affected. Poorly formed enamel easily breaks off or doesn't form at all leaving underlying dentin exposed. Likely affects both primary and permanent dentition.

Detection and diagnosis can occur as early as the eruption of primary and permanent teeth (Figures 29 and 30).
Figure 18. Typical case of Advanced Nursing Mouth Decay. Note the Lack of Involvement of the Mandibular Teeth. In Almost all Cases, the Tongue Covers and Protects Exposure of the Lower Teeth to Milk or Sweetened Substances When Breast or Bottle Fed
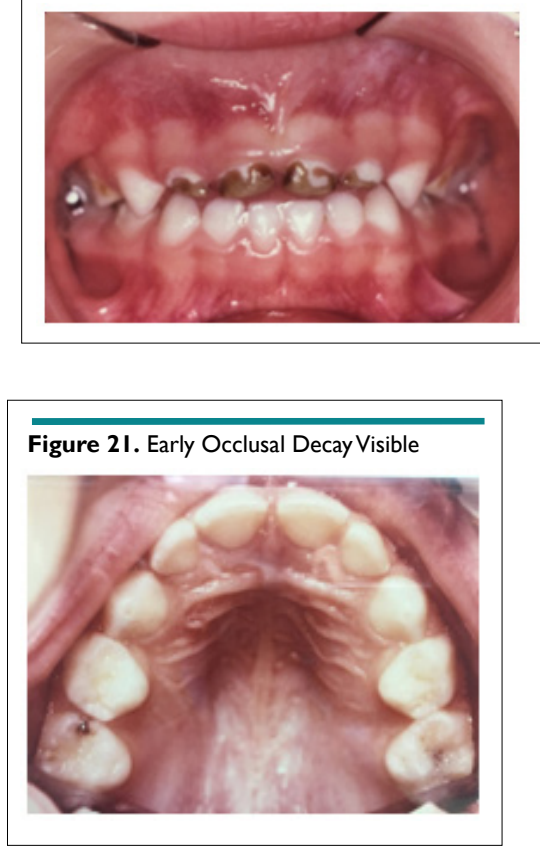
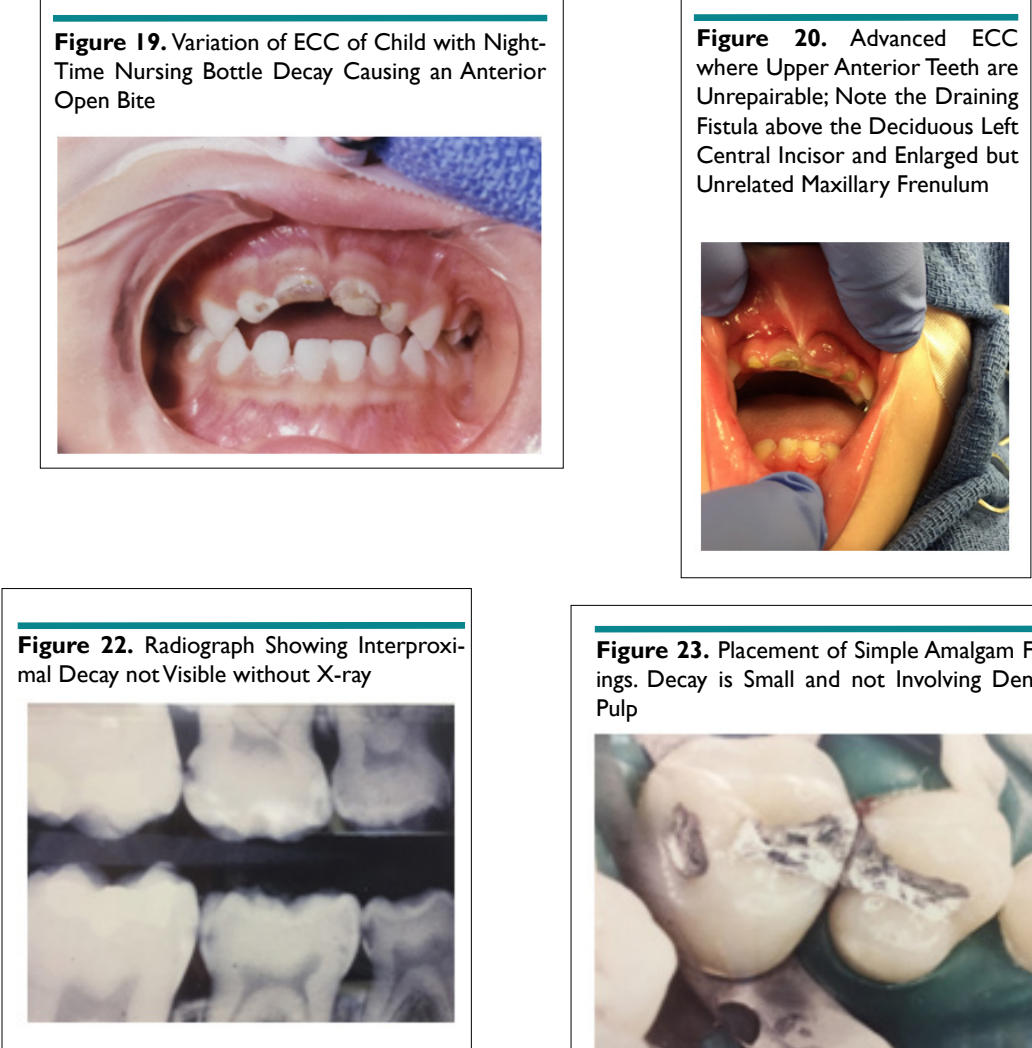

Figure 23. Placement of Simple Amalgam Fillings. Decay is Small and not Involving Dental Pulp

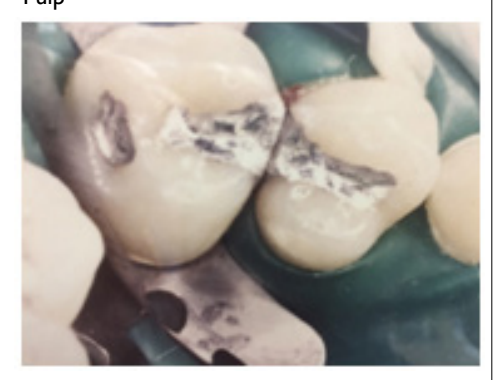




\section{Openventio
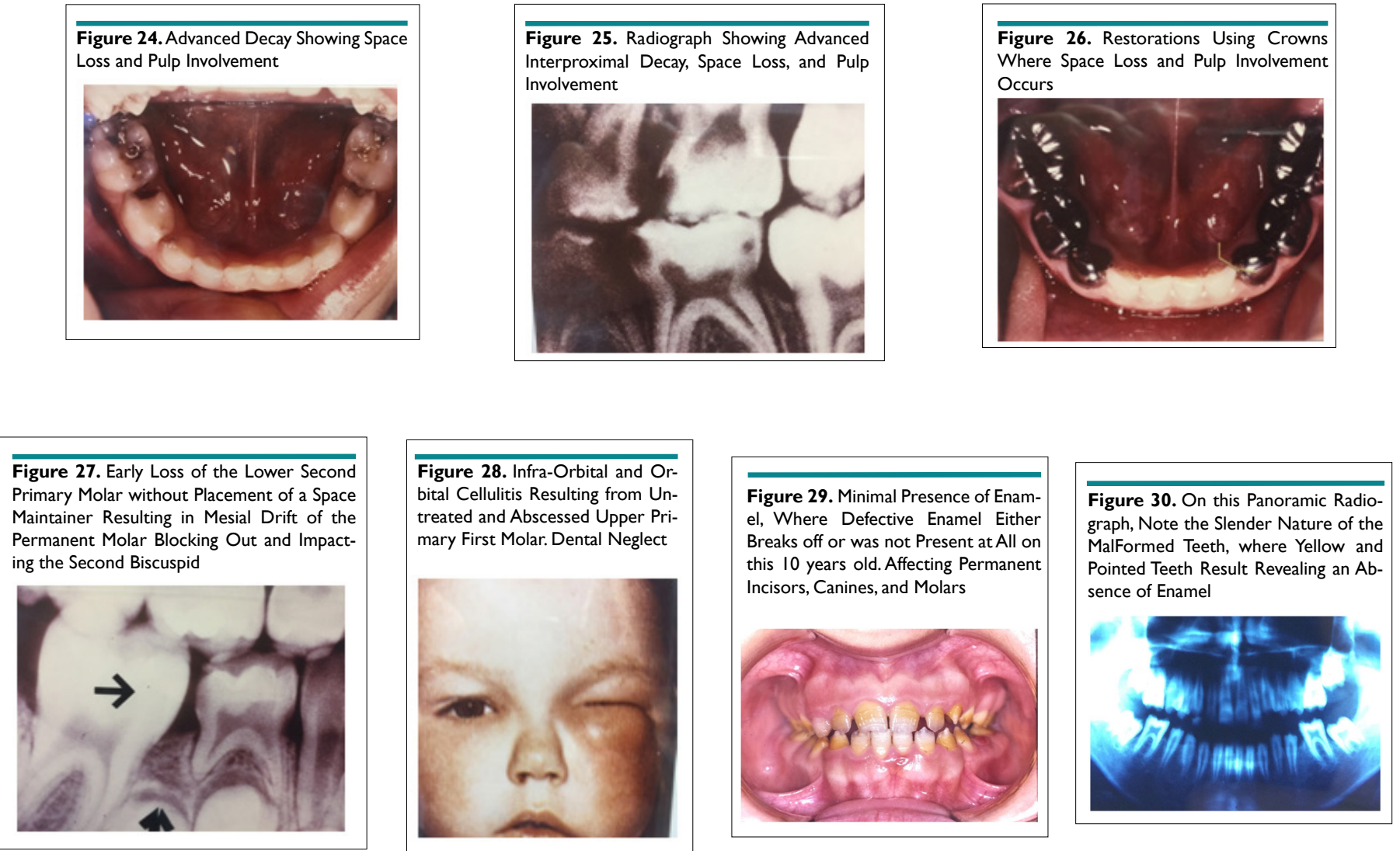

\begin{tabular}{|cccc|}
\hline \multicolumn{2}{|c|}{ Table I. Criteria for Fluoride Supplementation on basis of Age and amount of Fluoride in Drinking Water } & \\
\hline $\begin{array}{c}\text { F- content of drinking water } \\
\text { (ppm) }\end{array}$ & $\begin{array}{c}\mathbf{2} \text { weeks to } \mathbf{2} \text { years } \\
\mathbf{( m g )}\end{array}$ & $\begin{array}{c}\mathbf{2} \text { to } \mathbf{3} \text { years } \\
\text { ( } \mathbf{m g})\end{array}$ & $\begin{array}{c}\mathbf{3 - 1 4} \text { years } \\
\text { (mg) }\end{array}$ \\
\hline Less than 0.3 & 0.25 & 0.50 & 1.00 \\
\hline 0.3 to 0.7 & 0 & 0.25 & 0.50 \\
\hline Over 0.7 & $\begin{array}{c}\text { Dietary supplements } \\
\text { unnecessary }\end{array}$ & $\begin{array}{c}\text { Dietary supplements un- } \\
\text { necessary }\end{array}$ & $\begin{array}{c}\text { Dietary supplements } \\
\text { unnecessary }\end{array}$ \\
\hline
\end{tabular}

\section{Dentinogenesis Imperfecta}

Hereditary defect in which underlying dentin formation is aberrant; teeth take on a graying discoloration showing through an abnormal enamel shell. Seen in osteogenesis imperfecta also (blue sclera and weakened bone formation) (Figure 31).

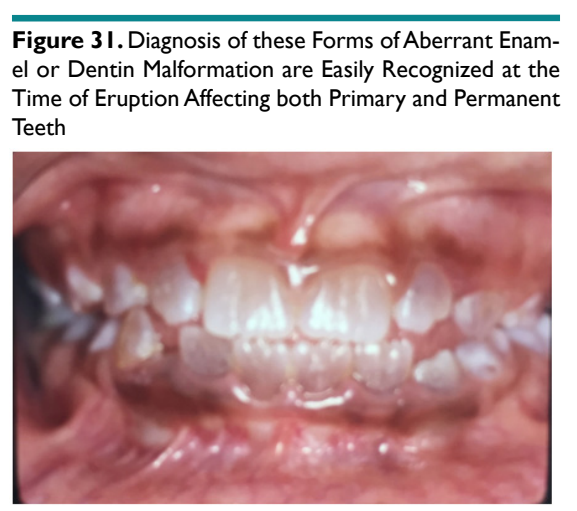

\section{Osteogenesis Imperfecta (Figure 32)}

Figure 32. Osteogenesis Imperfecta - A Deformity Com prised of Defects in Bone and Dentin Similar to Dentinogenesis Imperfecta

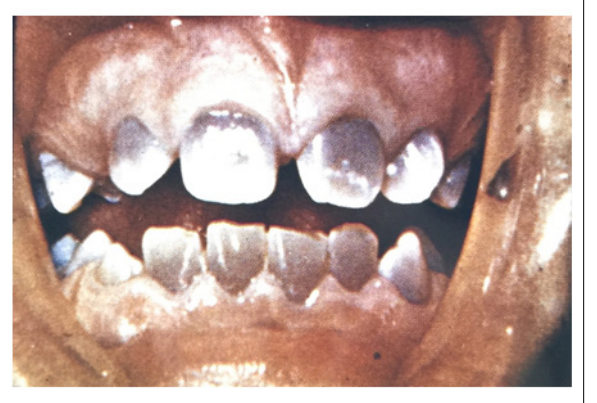




\section{ABERRATION IN ERUPTION AND EXFOLIATION OF} PRIMARY TEETH

Very rarely observed but found to manifest in the following diseases and local dental conditions

1. Hypophosphatasia

2. Non-Lipid Reticuloendothelial diseases (Eosinophilc granuloma, LettereSiwe, and Hand-Schuler)

3. Juvenile periodontitis

4. Down Syndrome

5. Presence of supernumerary teeth, congenitally missing tooth (or teeth)

6. Ectopic eruption of incisors or molars

\section{OCCLUSAL DISHARMONIES}

Discrepancies from normal with respect to eruption, alignment, settling of primary teeth can occur with numerous manifestations. Most common among undesirable positioning are the development of anterior and /or posterior dental cross bites. Narrow maxillary arches showing unilateral or bilateral nature seem to have a genetic heritage, but can also result from digital habits such as thumb or finger sucking, prolonged use of anatomically mis-shaped pacifiers, or bottle nipples most commonly resulting in anterior open bites. These malformations are temporary and in general easily managed by orthodontic intervention.

Crossbites can occur in the anterior maxilla, or posteriorly, in both unilateral or bilateral relationships. Orthodontic intervention is generally not recommended until ages 6-7 years; decisions to treat early generally reflect a high level of patient cooperation. Generally, however, most practitioners choose to defer until the permanent molars have emerged to provide greater anchorage for corrective measures.

\section{SUMMARY}

Discussion and inclusion of the above conditions and anomalies represents a limited but not uncommon sample of normality and abnormalities that manifest during the newborn and first two years of life.

While dental evaluations do not readily occur before the recommended first year, referral for pediatric dental consult is available when indicated. Early recognition of dental abnormalities, early onset of dental caries, and implementation of preventive strategies by medical personnel can set the stage for optimal oral health.

All photos and clinical conditions depicted in this manuscript were taken and treated by this author.

\section{REFERENCES}

1. Periodicity Schedule. American Academy of Pediatrics. 2002.

2. Periodicity Schedule, American Academy of Pediatrics, 2010.

3. Volker JF, Russell DL. The prevention of dental caries with fluoride, In: Finn SB, ed. Clinical Pedodontics. $4^{\text {th }}$ ed. St. Louis, MO,USA: WB Saunders; 1973: 494-506.

4. Clarkson B, Wei S. Fluorides in dental health. In: Stewart R, Barber T, Troutman K, Wei S, eds. Textbook of Pediatric Dentistry. St. Louis, MO, USA: WB Saunders; 1972: 717-747. 